Published in Revue médicale suisse, 2019, vol. 15, no. 650, pp. 947-950, which should be cited to refer to this work.

Eco-conception du cabinet de médecine de famille : concepts et vision d'avenir

Titre anglais: Eco-design of the general practice: concepts and forthcoming vision

Nombre de signes de l'article, espaces et ponctuation compris, mais sans compter les résumés, les implications pratiques, les figures, tableaux et références : 11988

\title{
Coordonées des auteurs :
}

Luc Ka Sing Ho (auteur principal)

Unisanté, Centre universitaire de médecine générale et santé publique, Lausanne Rue du Bugnon 44

1011 Lausanne

Tél : 0763219432 / 0795568335

luc.ho@chuv.ch

Yolanda Mueller

Unisanté, Centre universitaire de médecine générale et santé publique, Lausanne 1011 Lausanne

jolanda.mueller@hospvd.ch

François Héritier

Unisanté, Centre universitaire de médecine générale et santé publique, Lausanne 1011 Lausanne

francois.heritier@vtxnet.ch

Nicolas Senn

Unisanté, Centre universitaire de médecine générale et santé publique, Lausanne nicolas.senn@hospvd.ch

Julien Boucher, PhD

EA \& Haute école spécialisée de Suisse Occidentale (HES-SO/HEIG-VD)

julien.boucher@heig-vd.ch 


\section{Résumé français}

L'éco-conception est un terme relativement nouveau pour les cabinets de médecine de famille. Il s'agit d'une démarche pro-active visant à réduire les impacts environnementaux nuisibles engendrés par une organisation ou un produit.

Ces impacts environnementaux, comme le dérèglement climatique, représentent clairement des problèmes majeurs de santé publique, il semble donc légitime que le milieu médical s'interroge sur son rôle à "prévenir " plutôt que "guérir ", également via sa contribution à la protection de l'environnement.

Après avoir posé quelques éléments de définitions et de situations, cette revue dresse un état des lieux global de la pratique éco-conception dans le domaine médical ambulatoire. Enfin, des pistes de réflexions et de progrès sont présentées, avec un focus sur le contexte suisse.

\section{Résumé anglais}

Eco-design is relatively new to the family medicine practice. It consists of a pro-active approach in order to reduce harmful environmental impacts from an organization or a product.

These environmental impacts, such as climate change, clearly represent major public health concerns. It seems legitimate for the medical community to question its role in "preventing" rather than "curing" through its contribution to the protection of the environment.

This review lays some definitions and explains the current situation. It tries to gather the existing ecodesign initiatives in the outpatient area. At last, some lines of thought and of progress are presented, with a focus on the Swiss context.

\section{Introduction}


Les dégâts sur l'environnement causés par les gaz à effet de serre sont clairement établis. De plus, ceux-ci constituent un enjeu de santé publique majeur. En effet, l’Organisation Mondiale de la Santé (OMS) met en avant, dans une publication en 2018 , l'impact de la dégradation de l'environnement sur la santé de la population (1). Parmi les pathologies aggravées par le dérèglement climatique, nous retrouvons l'accroissement de la prévalence de certaines maladies à transmission vectorielle (fièvre Dengue, malaria, ...) mais également des pathologies respiratoires et cardiovasculaires (figure 1) (2).

\section{Figure 1}

Selon l'agence de protection de l'environnement aux Etats-Unis, I'utilisation énergétique du secteur des soins de la santé est classée en deuxième rang après l'industrie alimentaire (3). Les hôpitaux y produisent un volume de déchets de plus de 5.9 millions de tonnes par année (4), altérant directement l'environnement de notre planète avec des conséquences progressives sur la santé. Cette pollution contribue directement à environ $8 \%$ de production de gaz à effets de serre aux Etats-Unis (5). En comparaison avec l'aviation civile, ces mêmes émissions ne s'élèvent qu'à 3\% (6).

Dans ce cadre, il nous semble essentiel de s'interroger sur les impacts environnementaux générés par les cabinets médicaux. En effet, une pratique médicale de qualité devrait également se soucier des effets secondaires qu'elle peut avoir sur l'environnement. In fine, cela revient, indirectement, à agir sur la santé de la population. De plus, dans le contexte d'une demande croissante pour des produits et des services plus environnementalement et socialement responsables, les médecins sont invités à adapter leur pratique et de montrer, le cas échéant, leur implication dans cette problématique globale.

\section{Définition et utilité de l'éco-conception}

L'éco-conception correspond à la mise en œuvre de solutions préventives pour minimiser les impacts environnementaux d'un produit ou d'un service tout au long de son cycle de vie (figure 2) (7). Elle 
respecte les principes du développement durable et se distingue ainsi des approches environnementales classiques consistant à traiter, a posteriori, la pollution et les déchets une fois générés.

Figure 2

On pourra par exemple concevoir un produit qui utilise moins de matières premières, qui est conçu d’emblée pour être recyclé ou encore un produit plus léger ou plus petit pour limiter les impacts liés à la logistique (figure 3). De nombreuses stratégies sont possibles et il s'agit de trouver la plus adaptée à un contexte donné.

Figure 3

L'éco-conception doit avant tout être envisagée comme une démarche gagnant-gagnant qui allie la réduction des impacts environnementaux à la création de valeur d'usage (produits mieux adaptés aux utilisateurs) et de valeur économique (produit ou activité plus rentable dans une logique de coût complet). Les approches d'éco-conception se sont développées dans l'industrie ces dernières années, tant dans le cadre de produits physiques que dans le secteur des services. Toutefois dans le domaine médical, elles sont encore balbutiantes.

Afin d'amorcer le développement des bonnes pratiques, l'objectif de cet article est de réaliser une revue des initiatives existantes, ainsi que de proposer des méthodes pour une implémentation en Suisse.

Il s'agit à la fois d'identifier les éléments pratiques que le cabinet peut contrôler directement (par exemple la gestion des déchets sur site) ou influencer indirectement (par exemple la mobilité des patients), mais également de fournir les fondements scientifiques pour construire une compréhension commune des enjeux par les différents acteurs concernés.

\section{Ecologie au cabinet médical : état des lieux}


La fondation Global Green and Healthy Hospitals, basée sur plusieurs continents et comprenant plus de 36'000 hôpitaux membres, a façonné un guide destiné aux acteurs de la santé (8). Ce document publié en 2011 traite de l'impact environnemental sur la santé et présente dix objectifs à viser. Bien que son contenu soit principalement destiné aux hôpitaux, certaines thématiques comme la prise en compte des transports, de la chaîne alimentaire ou encore des achats de produits durables sont aussi bien applicables en cabinet.

Au Royaume-Uni, deux médecins généralistes publient en 2015 une liste des meilleurs conseils à appliquer afin d'optimiser la durabilité environnementale des soins primaires (9). Cette ressource a été développée dans le cadre d'un programme d'éducation médicale fournie par le Centre for Sustainable Healthcare, organisation britannique connue pour sa recherche sur la pratique d'un système de santé durable. Elle propose notamment des stratégies dans le domaine environnemental avec pour but l'amélioration de la santé.

L'Etat de Floride, aux Etats-Unis, a pour sa part mis en place un label écologique intitulé My Green Doctor pour les médecins s'engageant à promouvoir les gestes visant à améliorer la durabilité de leur pratique, puis à informer leurs patients sur les questions d'environnement. Cette inscription leur permet d'afficher ouvertement leur préoccupation et leur sens de la responsabilité. La délivrance de ce certificat, approuvé par la World Medical Association, passe par une concentration de plus de 140 étapes allant de l'éco-conception du cabinet à la façon de s'alimenter (10).

Ces pistes, bien qu'elles manquent parfois de substance, pourraient alimenter les discussions en Suisse. A noter que l'association de médecins en faveur de l'environnement propose également plusieurs moyens concrets pour diminuer l'impact écologique du cabinet (11). En l'absence de modèle suisse de pratique médicale centré sur la durabilité environnementale, nous sommes amenés à nous inspirer de conseils et de recommandations existant à l'étranger, souvent pour les hôpitaux, ainsi que de conseils généraux destinés à tout un chacun. 


\section{Applications pratiques d'une réflexion d'éco-conception}

Naturellement, nous pourrions penser que contribuer à la durabilité relève surtout du bon sens, mais celui-ci peut être trompeur en l'absence de données scientifiques. Cette complexité résulte notamment de la nature fortement multicritère des enjeux imbriqués dans les décisions à prendre. Afin de limiter les zones d'incertitude, des analyses doivent être menées pour pouvoir proposer une recommandation à l'utilisateur.

Dans le domaine spécifique du cabinet médical, la question du matériel à usage unique est fréquemment soulevée. En effet, un grand nombre d'instruments médicaux sont stérilisables à l'aide d'un autoclave (9). Dans ce contexte, il s'agit de tenir compte du niveau de rentabilité d'un matériel jetable et de l'empreinte des ressources utilisées, à mettre en balance avec le risque potentiel lié au non-respect de toutes les conditions d'asepsie.

Des études ont montré qu'en milieu hospitalier le processus de stérilisation, additionné à l'achat de matériels réutilisables, s'avère plus favorable que du matériel jetable seul (12-14). Cependant, selon la source d'énergie employée, la production de dioxyde de carbone peut être parfois plus importante et l'utilisation en ressource en eau peut être jusqu'à trois fois plus importante (13). D'un point de vue financier, l'investissement dans la stérilisation de matériel en 2008 pour un quart de tous les hôpitaux aux Etats-Unis a permis une économie annuelle de plus de 138 millions USD avec une réduction de déchets médicaux d'environ 2000 tonnes (15).

Dans un contexte ambulatoire cependant, les conséquences financières sont très différentes de I'hôpital. Les coûts d'un autoclave, de ses accessoires, de son entretien et de sa certification sont souvent des critères dissuasifs, d'autant plus si un seul médecin l'exploite. Sa rentabilité dépend également de la fréquence de son utilisation, de la disponibilité du personnel et de sa consommation en électricité. Des médecins généralistes installés au Royaume-Uni en ont fait la mauvaise expérience et ont été refroidis par l'absence d'avantages significatifs, et ceci malgré une exploitation partagée du 
matériel entre plusieurs médecins (16). Cet exemple de la stérilisation montre l'importance de mener une analyse multifactorielle des bénéfices et inconvénients.

\section{Quelles pistes concrètes pour l'avenir ?}

Appliquer la perspective "cycle de vie " (figure 2) à l'activité d'un cabinet de médecine de famille serait particulièrement utile. L'éco-bilan d'un cabinet médical type permettrait d'évaluer avec plus de précision quels sont les postes d'impacts les plus significatifs et donc les actions les plus pertinents à mettre en œuvre. En effet, uniquement une approche globale et objective prenant en compte l'ensemble des facteurs, y compris les considérations financières, peut être à même de définir les mesures de durabilité optimales à intégrer. Sans être exhaustif, les exemples qui nous semblent importants de considérer pour une analyse approfondie seraient de revoir les possibilités de mobilité autour du cabinet (patients et personnel), les choix énergétiques (sources et économies), les décisions d'achat matériel et consommables, le passage au fonctionnement informatique du cabinet, la question de la stérilisation et des plastiques à usage unique, et le développement de la culture « choosing wisely » dans la prescription d'examens complémentaires et de traitements (17).

Cette démarche devrait se baser sur une enquête auprès des prestataires de plusieurs cabinets, afin de baser les recommandations sur un échantillon de pratiques et de contextes suffisamment représentatifs.

Ainsi, une analyse détaillée de l'impact environnemental des cabinets pourrait amener à l'élaboration d'un consensus sur les éléments fondamentaux à considérer dans leur éco-conception. Et pourquoi pas aboutir à la création d'un label au niveau helvétique avec pour but de promouvoir l'éco-conception du cabinet, afin d'initier un mouvement vers le développement durable du domaine médical ambulatoire. 
Finalement, en élargissant encore l'approche, nous sommes en mesure de nous demander s'il ne faudrait pas questionner notre rôle de promoteur d'un mode de vie éco-responsable des patients. En effet, comme nous l'avons vu, l'activité humaine a un impact important sur l'environnement qui a luimême une influence importante sur la santé humaine. Certaines recommandations vont d'ailleurs dans ce sens, en mettant en avant l'importance d'une alimentation équilibrée ou d'une mobilité douce, en bref un environnement sain pour un corps sain !

\section{Conclusion}

Il n'est plus à démontrer que le dérèglement climatique est un enjeu majeur de santé publique et place la pratique médicale au cœur de cette problématique. Avec une approche d'éco-conception des cabinets médicaux, il est possible d'envisager de créer un espace de travail qui s'inscrit mieux dans la durabilité environnementale. Lors de son mandat en 2014, Margaret Chan, alors directrice générale de l'OMS, a mis l'accent sur le fait que nous devons nous convaincre que l'être humain est la plus importante des espèces menacées par le changement climatique. Devant ce changement de paradigme, le médecin de famille est invité à participer activement au maintien de la santé de notre planète comme de celle de nos patients.

\section{Déclaration de conflit d'intérêts}

Les auteurs n'ont déclaré aucun conflit d'intérêts en relation avec cet article. Julien Boucher est directeur du pôle éco-conception suisse, EA - Shaping Environmental Action, et chargé de recherche au sein de la Haute école spécialisée de Suisse occidentale (HES-SO/HEIG-VD).

\section{Stratégie de recherche dans Medline}


Les données utilisées pour cette revue ont été identifiées en partie par une recherche Medline des articles publiés en anglais ou en français depuis 2007 dans le domaine des impacts de l'environnement sur la santé et de la durabilité dans le domaine médical. Les principaux mots-clés utilisés pour la recherche étaient "climate change ", "carbon footprint", " environment ", "sustainable healthcare » et « life cycle assessment ».

\section{Implications pratiques}

-Le dérèglement climatique est un problème de santé publique majeur.

-Le médecin de famille est un acteur important pour sensibiliser la population générale à la problématique environnementale.

-Des initiatives dites « vertes » sont déjà appliquées à l'étranger, et nous pouvons nous y inspirer afin d'établir une ligne de bonne pratique en Suisse.

-L'éco-conception peut s'appliquer dans un cabinet de médecine de famille mais il peut être judicieux de faire appel à un spécialiste dans le domaine.

\section{Bibliographie}

1. World Health Organization. Climate change and health 2018 [cité le 10.12.2018 10]. Disponible sur: https://www.who.int/en/news-room/fact-sheets/detail/climate-change-and-health.

2.** Centers for Disease Controle and Prevention. Climate Effects on Health 2014 [cité le 12.12.2018].

3. Crowley RA, for the H, Public Policy Committee of the American College of P. Climate change and health: A position paper of the american college of physicians. Annals of Internal Medicine. 2016;164(9):608-10. doi: 10.7326/M15-2766. 
4.* Practice Greenhealth. Climate and Health 2018 [cité le 12.12.2018]. Disponible sur: https://practicegreenhealth.org/topics/climate-and-health.

5. Chung JW, Meltzer DO. Estimate of the carbon footprint of the us health care sector. JAMA. 2009;302(18):1970-2. doi: 10.1001/jama.2009.1610.

6. United States Environmental Protection Agency (EPA). Regulations for Greenhouse Gas Emissions from Aircraft 2017 [cité le 20.12.2018]. DIsponible sur: https://www.epa.gov/regulationsemissions-vehicles-and-engines/regulations-greenhouse-gas-emissions-aircraft.

7. Prendeville S. Envisioning Ecodesign: Definitions, Case Studies and Best Practices 2014.

8.** Global Green and Healthy Hospitals. A Comprehensive Environmental Health Agenda for Hospitals and Health Systems Around the World 2011 [cité le 08.02.2019]. Disponible sur: http://greenhospitals.net/wp-content/uploads/2011/10/Global-Green-and-Healthy-HospitalsAgenda.pdf.

9.* $\quad$ Centre for Sustainable Healthcare. Go Green for 2015 - Top Tips for General Practice Teams 2014 [cité le 09.12.2018]. Disponible sur: https://sustainablehealthcare.org.uk/news/2014/12/gogreen-2015-top-tips-general-practice-teams.

10. mygreendoctor.org. Green Doctor Office Recognition 2017 [cité le 12.12.2018].

11. Médecins en faveur de l'environnement. Ecologie au cabinet médical 2010 [cité le 19.12.2018]. Disponible sur: http://www.aefu.ch/fileadmin/user_upload/aefudata/b_documents/themen/praxisoekologie/ECM-Guide_Check-up_\%C3\%A9nerg\%C3\%A9tique.pdf.

12. Kwakye G, Pronovost PJ, Makary MA. Commentary: A Call to Go Green in Health Care by Reprocessing Medical Equipment. 2010;85(3):398-400. doi: 10.1097/ACM.0b013e3181cd5a71. PubMed PMID: 00001888-201003000-00010. 
13. McGain F, Story D, Lim T, McAlister S. Financial and environmental costs of reusable and single-use anaesthetic equipment. $\mathrm{Br} J$ Anaesth. 2017;118(6):862-9. Epub 2017/05/16. doi: 10.1093/bja/aex098. PubMed PMID: 28505289.

14. Eckelman M, Mosher M, Gonzalez A, Sherman J. Comparative life cycle assessment of disposable and reusable laryngeal mask airways. Anesth Analg. 2012;114(5):1067-72. Epub 2012/04/12. doi: 10.1213/ANE.0b013e31824f6959. PubMed PMID: 22492190.

15. Kwakye G, Brat GA, Makary MA. Green surgical practices for health care. Archives of Surgery. 2011;146(2):131-6. doi: 10.1001/archsurg.2010.343.

16.* GPonline. To sterilise or dispose? That is the question 2007 [cité le 21.12.2018]. Disponible sur: https://www.gponline.com/sterilise-dispose-question/article/658486.

17. Regard S, Gaspoz JM, Kherad O. ["Less is more"]. Revue medicale suisse. 2013;9(381):770, 24. Epub 2013/05/11. PubMed PMID: 23659155.

18. Boucher J E, P, Damien F et al,. Life Cycle Perspective in ISO14001 : a game changer - How to increase your performance with ecodesign? 2017 [cité le 20.02.2019]. Disponible sur: http://cloud2.snappages.com/b0d6d10923becba07c0287d0b0af8fd47ed8a57d/Brochure_ENEC2017-online_1.pdf.

\section{Figures, tableaux et annexes}




\section{Impact of Climate Change on Human Health}

\author{
Injuries, fatalities, \\ mental health impacts \\ Asthma,
cardiovascular disease
}

Air

Pollution

Heat-related illness

and death,

cardiovascular failure

Forced migration,

civil conflict,

mental health impacts

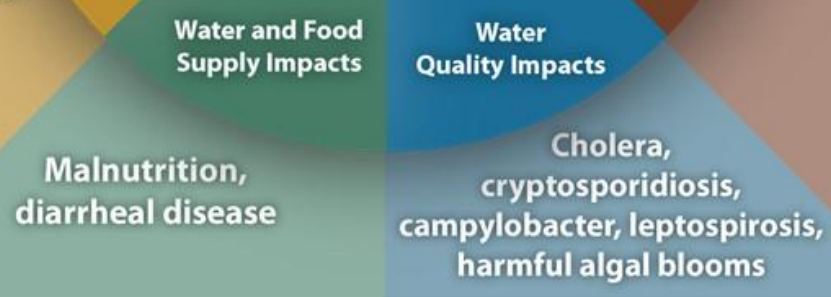

Malaria, dengue, encephalitis, hantavirus,

Rift Valley fever,

Lyme disease,

chikungunya,

Ecology West Nile virus

Increasing

Allergens Respiratory

allergies, asthma

Figure 1 : Les différents impacts du dérèglement climatique sur la santé humaine : alors que des nouvelles menaces émergent sur le plan sanitaire, les personnes âgées, les enfants de bas âge et les populations avec des ressources économiques plus limitées sont les premiers touchés. Diagramme tiré du site du $C D C$, destiné à l'usage publique (2).

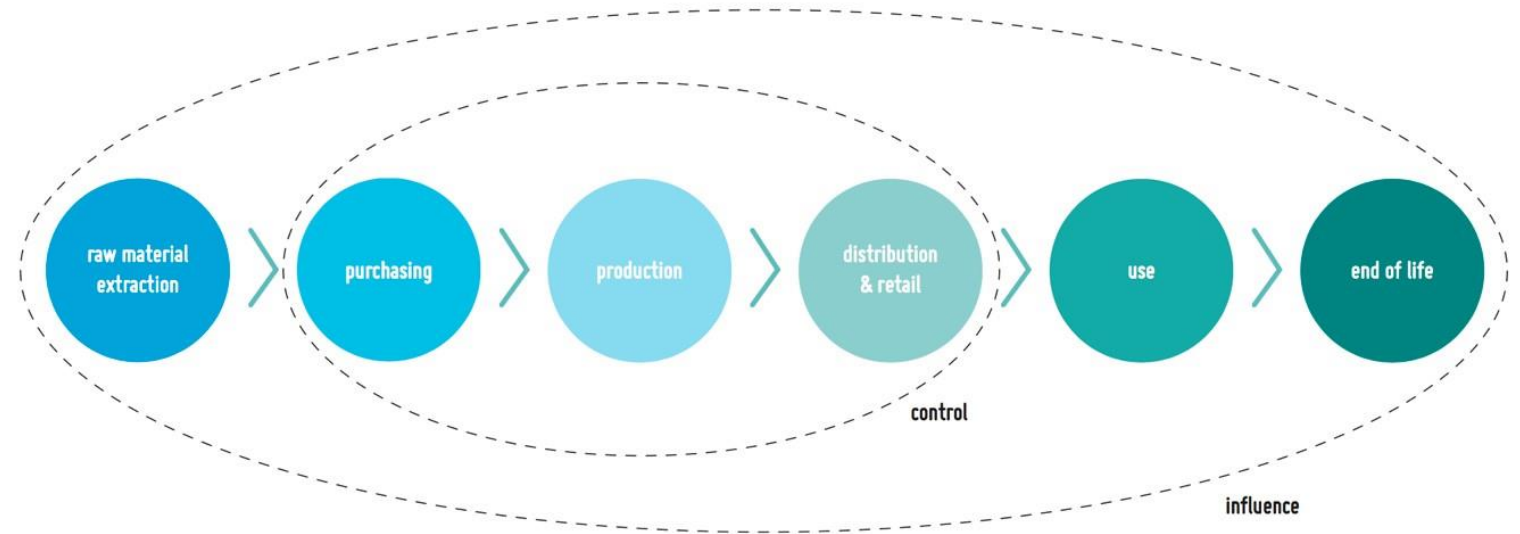


Figure 2 : Le cycle de vie d'un produit ou d'un service, dit « du berceau à la tombe ", et les zones de contrôle et d'influence de l'entreprise. On utilise le terme " responsabilité » étendue de l'entreprise pour indiquer que dans le cadre d'une démarche d'éco-conception, l'entreprise doit tout mettre en œuvre pour minimiser l'impact des étapes sous son contrôle direct et, dans la mesure du possible, influencer favorablement les autres étapes (18).

Concept du produit

Optimisation de la fin
de vie
- Réutilisation de produit
- Re-fabrication / réhabilitation
- Favoriser le recyclage et/ou
des fins de vie appropriées
Optimisation de la
durée de vie
- Faciliter le démontage et la
maintenance
- Durabilité et fiabilité
- Relation produit-utilisateur
forte

Structure du produit

Composants du produit

- Matériaux à faibles impacts (renouvelables, recyclables, recyclés, ...)

Développement de nouveaux concepts

- Utilisation partagée du produit

- Fonctionnalité optimisée

- Multifonction

- Dématérialisation

- Réduction du poids et du volume

- Dématérialisation

Optimisation des

techniques de fabrication

- Techniques alternatives

- Réduction des étapes de productions

- Consommation d'énergie renouvelable

- Réduction des déchets

- Réduction des consommables

Système du produit

Figure 3 : Principales stratégies d'éco-conception, inspirées de Life Cycle Perspective in ISO14001: a

game changer - How to increase your performance with ecodesign ? (18)

QCM

L'éco-conception d'un cabinet de médecine de famille consiste à :

-diminuer les coûts de la santé liés à la médecine de premier recours.

+respecter les principes du développement durable. 
-traiter des déchets et de la pollution générée par le cabinet.

-adopter une pratique médicale de manière à obtenir un meilleur rendement financier.

+analyser les cycles de vie des différents produits et services liés au cabinet afin de minimiser ses impacts environnementaux. 